\title{
Article
}

Subscriber access provided by ECU Libraries

\section{Dissecting the structural and chemical determinants of the 'open-to- closed' motion in the mannosyltransferase PimA from mycobacteria.}

Ane Rodrigo-Unzueta, Mattia Ghirardello, Saioa Urresti, Ignacio Delso, David Giganti, Itxaso Anso, Beatriz

Trastoy, Natalia Comino, Montse Tersa, Cecilia D'Angelo, Javier Orlando Cifuente, Alberto Marina, Jobst Liebau, Lena Mäler, Alexandre Chenal, David Albesa-Jove, Pedro Merino, and Marcelo E. Guerin

Biochemistry, Just Accepted Manuscript • DOI: 10.1021/acs.biochem.0c00376 • Publication Date (Web): 28 Jul 2020

Downloaded from pubs.acs.org on August 1, 2020

\section{Just Accepted}

"Just Accepted" manuscripts have been peer-reviewed and accepted for publication. They are posted online prior to technical editing, formatting for publication and author proofing. The American Chemical Society provides "Just Accepted" as a service to the research community to expedite the dissemination of scientific material as soon as possible after acceptance. "Just Accepted" manuscripts appear in full in PDF format accompanied by an HTML abstract. "Just Accepted" manuscripts have been fully peer reviewed, but should not be considered the official version of record. They are citable by the Digital Object Identifier (DOI®). "Just Accepted" is an optional service offered to authors. Therefore, the "Just Accepted" Web site may not include all articles that will be published in the journal. After a manuscript is technically edited and formatted, it will be removed from the "Just Accepted" Web site and published as an ASAP article. Note that technical editing may introduce minor changes to the manuscript text and/or graphics which could affect content, and all legal disclaimers and ethical guidelines that apply to the journal pertain. ACS cannot be held responsible for errors or consequences arising from the use of information contained in these "Just Accepted" manuscripts. 
Dissecting the structural and chemical determinants of the 'open-to-closed' motion in the mannosyltransferase PimA from mycobacteria.

\section{Ane Rodrigo Unzueta ${ }^{1,2, \#, \text { Mattia Ghirardello }}{ }^{3, \#}$, Saioa Urresti, ,2,\#, Ignacio Delso ${ }^{3}$, David Giganti $^{1,2,4}$, Itxaso Anso ${ }^{5}$, Beatriz Trastoy ${ }^{5}$, Natalia Comino ${ }^{5}$, Montse Tersa ${ }^{5}$, Cecilia D'Angelo5, Javier O. Cifuente ${ }^{5}$, Alberto Marina ${ }^{5}$, Jobst Liebau ${ }^{6}$, Lena Mäler6,7, Alexandre Chenal ${ }^{8}$, David Albesa-Jové, ${ }^{1,2,5,9}$, Pedro Merino ${ }^{10, *}$, and Marcelo E. Guerin ${ }^{1,2,5,9, *}$}

${ }^{1}$ Instituto Biofisika, Centro Mixto Consejo Superior de Investigaciones Científicas Universidad del País Vasco/Euskal Herriko Unibertsitatea (CSIC,UPV/EHU), Barrio Sarriena s/n, Leioa, Bizkaia, 48940, Spain,

${ }^{2}$ Departamento de Bioquímica, Universidad del País Vasco, Barrio Sarriena s/n, Leioa, Bizkaia, 48940, Spain,

${ }^{3}$ Department of Synthesis and Structure of Biomolecules. Institute of Chemical Synthesis and Homogeneus Catalysis (ISQCH). University of Zaragoza-CSIC. 50009 Zaragoza, Spain,

${ }^{4}$ Unité de Microbiologie Structurale (CNRS URA 2185), Institut Pasteur, 25 rue du Dr. Roux, 75724, Paris Cedex 15, France,

${ }^{5}$ Structural Biology Unit, CIC bioGUNE, Bizkaia Technology Park, 48160 Derio, Spain,

${ }^{6}$ Department of Biochemistry and Biophysics, Stockholm University, 10691 Stockholm, Sweden,

${ }^{7}$ Department of Chemistry, Umeå University, 90187 Umeå, Sweden

${ }^{8}$ Unité de Biochimie des Interactions Macromoléculaires (CNRS UMR 3528), Institut Pasteur, 25 rue du Dr. Roux, 75724, Paris Cedex 15, France,

${ }^{9}$ IKERBASQUE, Basque Foundation for Science, 48013, Bilbao, Spain,

${ }^{10}$ Glycobiology Unit. Institute of Biocomputation and Physics of Complex Systems (BIFI). University of Zaragoza. Campus San Francisco. 50009 Zaragoza, Spain.

*To whom correspondence should be addressed: Marcelo E. Guerin, Structural Biology Unit, CIC bioGUNE, Bizkaia Technology Park, 48160 Derio, Spain, mrcguerin@cicbiogune.es; Pedro Merino, Glycobiology Unit. Institute of Biocomputation and Physics of Complex Systems (BIFI). University of Zaragoza. Campus San Francisco, 50009 Zaragoza, Spain, pmerino@unizar.es.

\# These authors equally contributed to this work.

Keywords: carbohydrate-modifying enzyme, glycosyltransferase, conformational changes, structural biology, X-ray crystallography 


\begin{abstract}
The phosphatidyl-myo-inositol mannosyltransferase A (PimA) is an essential peripheral membrane glycosyltransferase that initiates the biosynthetic pathway of phosphatidyl-myoinositol mannosides (PIMs), key structural elements and virulence factors of Mycobacterium tuberculosis. PimA undergoes functionally important conformational changes, including (i) $\alpha$-helix-to- $\beta$-strand and $\beta$-strand-to- $\alpha$-helix transitions, and (ii) an 'open-to-closed' motion between the two Rossmann-fold domains, a conformational change necessary to generate a catalytically competent active site. In previous work, we established that GDP-Man and GDP stabilize the enzyme and facilitate the switch to a more compact active state. To determine the structural contribution of the mannose ring in such activation mechanism we analyzed a series of chemical derivatives, including mannose-phosphate (Man-P) and mannose-pyrophosphateribose (Man-PP-RIB), and additional GDP derivatives, as pyrophosphate-ribose (PP-RIB) and GMP, by the combined used of X-ray crystallography, limited proteolysis, circular dichroism, isothermal titration calorimetry and Small Angle X-ray Scattering methods. Although the $\beta$ phosphate is present, we found that the mannose ring, neither covalently attached to phosphate (Man-P) nor to PP-RIB (Man-PP-RIB), does promote the switch to the active compact form of the enzyme. Therefore, the nucleotide moiety of GDP-Man, and not the sugar ring, facilitates the 'open-to-closed' motion, with the $\beta$-phosphate group providing the high affinity binding to PimA. Altogether, the experimental data, contribute to a better understanding of the structural determinants involved in the 'open-to-closed' motion observed not only in PimA, but also visualized/predicted in other glycosyltransfeases. In addition, the experimental data might prove useful for the discovery/development of PimA and/or glycosyltransferase inhibitors.
\end{abstract}




\section{INTRODUCTION}

It is now generally accepted that proteins do not just occupy one single state and biological functions, i.e. substrate recognition, binding, stabilization of transition states and product release, are ultimately rooted in the coordinated physical motions of the atoms, localized protein regions, motifs, and/or protein domains in a wide range of timescales. Protein dynamics can be defined as any time-dependant change in atomic coordinates including both equilibrium fluctuations and non-equilibrium changes (1). A hypothesis proposed over 30 years ago by Frauenfelder and colleagues and more recent experimental data suggest that protein undergoes conformational changes leading to an ensemble of conformations sampled over the time in the multidimensional energy landscape $(1,2,3,4)$. The landscape defines the amplitude and directionality, and the timescale of the motions to adopt substates (1). Therefore, understanding the relationship between structure, catalysis and function of proteins often requires the consideration of their dynamic behavior/nature, understood as the ensemble of conformations sampled in a multidimentional/intricate energy landscape. These intrinsic conformational transitions can be classified by the amplitude of motions. Submicrosecond fluctuations arising from side chains rotations and small loops movements are commonly observed during substrate recognition and catalysis. In addition, enzyme function can involve slower and larger-amplitude collective motions, as fold-switches or domain motions, between a relatively small number of substates (5). Correlations between the conformational fluctuation timescale and the catalytic turnover have been empirically stablished for a variety of enzymes - e.g. CypA (3) and HIV-1 protease (6). Interestingly, the rate of conformational change has been shown to coincide with the rate limiting step, such as product release (3).

The phosphatidyl-myo-inositol mannosyltransferase A (PimA), is an essential membrane glycosyltransferase (GT) that initiates the biosynthesis of phosphatidyl-myoinositol mannosides (PIMs), lipomannan (LM) and lipoarabinomannan (LAM), key structural 
elements and virulence factors of Mycobacterium tuberculosis $(7,8,9)$. PimA catalyzes the transfer of a Manp residue from GDP-Man to the 2-position of phosphatidyl-myo-inositol (PI) to form phosphatidyl-myo-inositol monomannoside $\left(\mathrm{PIM}_{1}\right)$ on the cytoplasmic side of the plasma membrane (see the chemical structure of $\mathrm{Ac}_{2} \mathrm{PIM}_{6}$ and the scheme of the reaction catalyzed by PimA in Figure S1; 7, 10). PimA is an amphitropic GT that belongs to the GT-B superfamily and is composed of two Rossmann-fold domains separated by a central deep cleft that includes the catalytic center $(11,12,13,14)$. The unliganded crystal form of PimA presents four monomers in the asymmetric unit, capturing two different conformational states of the enzyme. The two states are structurally similar to each other, displaying an 'open' conformation. However, an $\alpha$-helical hairpin consisting of helices $\alpha 4$ (residues 134-145) and $\alpha 5$ (residues 149-157) folds back against the N-terminal domain in one conformational state (the 'compact inactive' state, thereafter), whereas in the other state it displays an extended conformation (the 'extended inactive' state, thereafter) that is partially disordered and protrudes away from the N-terminal domain core (Figure 1; 16, 17). The structural comparison of the unliganded form with that of PimA in complex with GDP-Man or GDP revealed important conformational changes. Specifically, (i) the occurrence of fold-switch $\beta$ strand-to- $\alpha$-helix and $\alpha$-helix-to- $\beta$-strand transitions in the N-terminal domain of the enzyme (residues 118-163; 15, 17), and (ii) an domain rearrangement from an 'open' ('active extended' state, thereafter) to a 'closed' ('active compact' state, thereafter) state $(11,18,19)$. Moreover, the fold-switching is a functional event that activates PimA, since a PimA mutant locked in both extended and compact unliganded conformations is inactive, while a mutant locked in the substrate-bound conformations is active (15). 


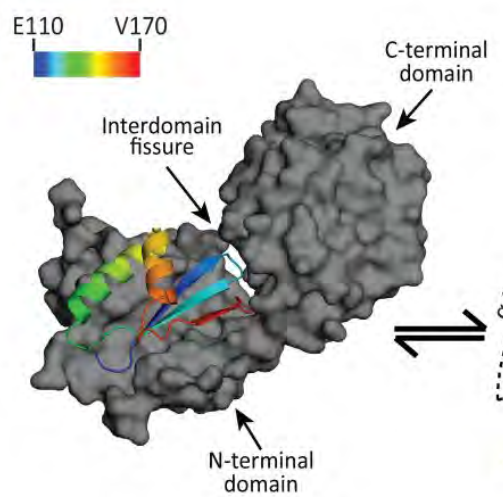

inactive compact 'open' unliganded form

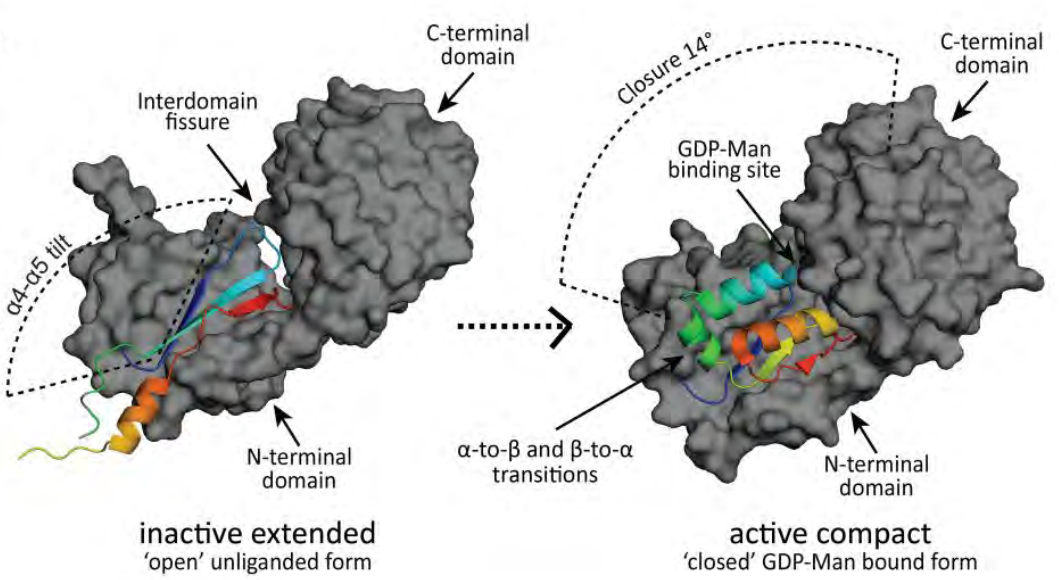

Figure 1. Current structural snapshots of PimA along the catalytic cycle. The crystal structure of the unliganded form of PimA (PDB ID 4NC9) trapped two different conformations of the enzyme, the 'inactive compact' and 'inactive extended'. An $\alpha$-hairpin moves away from the core of the N-terminal domain. The crystal structure of PimA in complex with GDP-Man (PDB ID 2GEJ) reflects the 'active compact' state of the enzyme. The transition to the active state involves a fold-switch event localized in a region of the N-terminal domain. It is worth noting that both inactive forms of PimA crystallized in an 'open' conformation with respect of the $\mathrm{N}$ - and C-terminal domains, whereas the active conformation crystallized in a 'closed conformation'.

The large amount of structural evidences places us in an unprecedented position to study the donor substrate-mediated mechanism of activation of PimA. Here we decipher the structural contribution of GDP-Man to the activation mechanism of PimA, by the combined use of nucleotide sugar chemistry, biochemistry, X-ray crystallography, small-angle X-ray scattering, circular dichroism and isothermal titration calorimetry. 


\section{EXPERIMENTAL PROCEDURES}

\section{Materials and Methods}

Guanosine 5'-diphosphate-D-mannose (GDP-Man), guanosine 5'-diphosphate (GDP), guanosine 5'-monophosphate (GMP), guanosine (GNO), guanine (GUA), mannose 1phosphate and elastase protease were purchased from Sigma. Recombinant PimA from $M$. smegmatis (MSMEG_2935) was produced in Escherichia coli and purified to apparent homogeneity as previously described $(15,18)$. The reaction flasks and other glass equipment were heated in an oven at $130{ }^{\circ} \mathrm{C}$ overnight and assembled in a stream of argon. All reactions were monitored by TLC on silica gel 60 F254; the positions of the spots were detected by $\lambda=254 \mathrm{~nm}$ UV light or by spraying with either a $5 \%$ solution of phosphomolybdic acid in ethanol or Mostain solution. Column chromatography was carried out in a Buchi 800 MPLC system or a Combiflash apparatus by using silica gel 60 microns and with solvents that were distilled prior to use. Melting points were uncorrected. Purification by semipreparative HPLC (column Atlantis ${ }^{\circledR}$ DC18 $5 \mu \mathrm{m}, 19 \times 100 \mathrm{~mm}$, flow: $12.5 \mathrm{~mL} \mathrm{~min}^{-1}$ ) was carried out in a Waters 515 pump system with photodiode array (PDA) detection. ${ }^{1} \mathrm{H}$ and ${ }^{13} \mathrm{C}$ NMR spectra were recorded on Bruker Avance $400 \mathrm{MHz}$ or AVANCE II $300 \mathrm{MHz}$ instruments in the stated solvent. Chemical shifts are reported in ppm $(\delta)$ relative to $\mathrm{CHCl}_{3}(\delta=7.26)$ in $\mathrm{CDCl}_{3}$. NMR assignments were made by using standard $2 \mathrm{D}$ experiments. Optical rotations were recorded on a JASCO DIP-370 polarimeter. Elemental analyses were performed on a PerkinElmer 240B microanalyzer or with a PerkinElmer 2400 instrument.

\section{MsPimA •GMP crystallization and Data Collection}

The best crystals of the $M s$ PimA $\bullet$ GMP form were obtained by mixing $2.0 \mu$ of GSGA-PimA $\left(10 \mathrm{mg} \mathrm{ml}^{-1}\right)$ in the presence of $1 \mathrm{mM} \mathrm{GMP}$, with $2.0 \mu \mathrm{l}$ of a mother liquor of $18 \%$ PEG 8000 , $200 \mathrm{mM}$ calcium acetate and $50 \mathrm{mM}$ HEPES pH 7.5. Crystals appeared after 2-3 days and 
grew as rods. Prior to data collection, crystals were transferred to a cryoprotectant solution (25\% glycerol in the mother liquor) and immediately cryo-cooled in liquid nitrogen. Crystals of $M s$ PimA $\cdot$ GMP belong to the orthorhombic space group $P 2_{1} 2_{1} 2_{1}$ and have 1 molecule per asymmetric unit, corresponding to a Matthews coefficient of $2.31 \AA^{3}$ and a solvent content of 46.7\%. X-ray diffraction data from a single crystal were collected on Proxima 1 beamline $(\lambda=$ $0.98011 \AA$ ) at Soleil (France) equipped with a CCD ADSC Quantum 315r system detector, and processed with program XDS (20). A total of 300 diffraction images were collected with a $0.5^{\circ}$ rotation between images.

\section{MsPimA •GMP structure determination and refinement}

The crystal structure of the $M s$ PimA•GMP complex was solved by molecular replacement method with the program Phaser and PHENIX suite $(21,22)$, using the atomic coordinates of MsPimA•GDP-Man complex as the search model (PDB ID 2GEJ: 11). Model rebuilding was carried out with the CCP4 suite (23). The final manual building was carried out with Coot (24) and refined with phenix.refine (25). The structure was validated by MolProbity (26). Data collection and refinement statistics are shown in Table S1. The atomic coordinates and structure factors have been deposited in the Protein Data Bank, (PDB ID 5BRS).

\section{Chemical synthesis of Man-PP-RIB and PP-RIB}

Please see Supporting Information for details.

\section{Limited Proteolysis of MsPimA}

Recombinant purified $M s$ PimA $(25 \mu \mathrm{g})$ was incubated with $0.10 \mu \mathrm{g}$ of elastase in $100 \mu \mathrm{l}$ of 50 $\mathrm{mM}$ Tris- $\mathrm{HCl} \mathrm{pH} 7.5$, in the presence of substrates for $0-90$ min at $37^{\circ} \mathrm{C}$. Aliquots of $12 \mu \mathrm{l}$ were mixed with $3 \mu \mathrm{l}$ of $250 \mathrm{mM}$ Tris- $\mathrm{HCl} \mathrm{pH}$ 6.8, 10\% SDS, 50\% glycerol, $500 \mathrm{mM}$ DTT 
and $0.01 \%$ bromophenol blue at the indicated times. Samples were boiled for 3 min and run onto a NuPAGE ${ }^{\circledR} 4-12 \%$ gel (Invitrogen). Protein bands were visualized by staining the gel with SimplyBlue ${ }^{\mathrm{TM}}$ SafeStain (Invitrogen).

\section{Near-UV Circular Dichroism analysis}

Spectra were adquired in a J-810 CD spectropolarimeter (Jasco Corp., Tokio, Japan) by using Hellma 105.200-QS quartz cuvettes with a $1 \mathrm{~cm}$ optical path. Spectra were recorded in a continuous mode with $1 \mathrm{~nm}$ band width, one second response and a scan speed of 100 $\mathrm{nm} / \mathrm{min}^{-1}$. Samples were $10 \mu \mathrm{M} M s \mathrm{PimA}$ in $10 \mathrm{mM}$ Tris-HCl $\mathrm{pH}$ 7.5. Substrates were added in a 1:10 ratio and 25 scans were accumulated to obtain the final spectra, which were further corrected for the baseline signal. Spectra were recorded in the $250-300 \mathrm{~nm}$ range at $20^{\circ} \mathrm{C}$.

\section{Temperature scans}

Spectra were adquired in a J-810 CD spectropolarimeter (Jasco Corp., Tokio, Japan) by using Hellma 110-QS quartz cuvettes with a $1 \mathrm{~mm}$ optical path, by using a Peltier thermal device, allowing the temperature control during the experiments. Spectra were recorded in a continuous mode with $1 \mathrm{~nm}$ band width, one second response and a scan speed of 100 $\mathrm{nm} / \mathrm{min}^{-1}$. Samples were $4.61 \mu \mathrm{M} M s \mathrm{PimA}$ in $10 \mathrm{mM}$ Tris-HCl $\mathrm{pH}$ 7.5. Substrates were added in a 1:10 ratio. In this case, thermal dependancies of the ellipticity were monitorized in the range from $10^{\circ} \mathrm{C}$ to $90^{\circ} \mathrm{C}$ at $222 \mathrm{~nm}$. Temperature was increased stepwise by $1 \% \mathrm{~min}$. (Table $1)$.

\section{Small Angle X-ray Scattering Measurements}

Synchrotron X-ray diffraction data for purified recombinant $M s$ PimA in its unliganded form and in the presence of GDP-Man, GDP, GMP, PP-RIB, Man-P and Man-PP-RIB were 
collected in batch mode. The sample volume loaded was $30 \mu \mathrm{L}(1.5 \mathrm{~mm}$ diameter capillary with $10 \mu \mathrm{m}$ wall thickness). Data were collected on a pixel Pilatus $2 \mathrm{M}$ detector at Diamond Light Source B21 beamline (UK). The scattering patterns were measured as an accumulation of frames taken as $18 \times 0.5$-sec exposures for protein samples at a minimum of three different protein concentrations ranging from 4 to $8 \mathrm{mg} / \mathrm{ml}$. To check for radiation damage, the 18 frames were compared as a time-series; no radiation damage was observed. Using the sampleto-detector distance of $3914 \mathrm{~mm}$, the range of momentum transfer values is $0.004<q<$ $0.37 \AA^{-1}(q=4 \pi \sin (\theta) / \lambda$ where $2 \theta$ is the scattering angle and $\lambda=1 \AA$ is the X-ray wavelength). Data were processed using standard procedures by the program packages ScÅtter (developed by Rob Rambo at Diamond Light Source) and PRIMUS (27). The forward scattering (I(0)) was evaluated using the Guinier approximation (28) assuming the intensity is represented as $I(q)=I(0) \exp \left(-\left(q R_{g}\right)^{2} / 3\right)$ for a very small range of momentum transfer values $\left(q<1.3 / R_{g}\right)$. The maximum dimensions $\left(D_{\max }\right)$, the interatomic distance distribution functions $(P(\mathrm{r}))$, and the radii of gyration $\left(R_{g}\right)$ were computed using GNOM (29) (see Table S2 for details).

\section{Isothermal titration calorimetry}

Ligand binding to MsPimA was assayed using the high precision VP-ITC system (MicroCal Inc.) as previously described (11) with the following modifications. The ITC cell $(1.4 \mathrm{ml})$ contained $10 \mu \mathrm{M} M s \mathrm{PimA}$ in $25 \mathrm{mM}$ Tris- $\mathrm{HCl} \mathrm{pH} 7.5$ and $150 \mathrm{mM} \mathrm{NaCl}$, and the syringe (150 $\mu \mathrm{l}$ ) contained $150 \mu \mathrm{M}$ of GDP, $500 \mu \mathrm{M}$ of GMP, Man-P, Man-PP-Rib or PP-Rib, in the same buffer. Sample solutions were thoroughly degassed under vacuum and each titration was performed at $25^{\circ} \mathrm{C}$ by one injection of $2 \mu \mathrm{l}$ followed by 29 injections of $10 \mu \mathrm{l}$ with 210 seconds between injections using a $290 \mathrm{rpm}$ rotating syringe. Raw heat signal collected with a $16 \mathrm{~s}$ filter was corrected for the dilution heat of the ligand in the PimA buffer and normalized 
to the concentration of ligand injected. Data were fit to a bi-molecular model using the Origin $^{\mathrm{TM}}$ software provided by the manufacturer. 


\section{RESULTS}

\section{The crystal structure of MsPimA in complex with guanosine 5'-monophosphate}

We were able to solve the crystal structure of PimA from Mycobacterium smegmatis (MsPimA; UniProt ID A0QWG6) in complex with guanosine 5'-monophosphate (GMP) at 2.6 Å resolution (Data collection and refinement statistics are shown in Table S1; Figure 2A). MsPimA crystallized in an active compact conformation, similar to that observed in the $M s$ PimA $•$ GDP (PDB ID 2GEK) and $M s$ PimA•GDP-Man (PDB ID 2GEJ) complexes (r.m.s.d. of $0.4 \AA$ for 361 equivalent residues). In contrast, the structural comparison of the $M s$ PimA $\bullet$ GDP complex with the inactive compact and extended unliganded forms of MsPimA revealed the local reshuffling of secondary structure elements within the flexible segment (residues 118-163) in the N-terminal domain (Figure 1; r.m.s.d. of $5.6 \AA$ and $9.2 \AA$ for 366 and 358 equivalent residues, respectively; 15). The GMP molecule is clearly visible in the electron density map, located at the interface between the $\mathrm{N}$ - and $\mathrm{C}$-terminal domains (Figure 2B and 2C; see the Final $(2 F o-F c)$ electron density map for $M s$ PimA-GMP complex (contoured at $1 \sigma$ ) in Figure S2). The guanidyl heterocycle binds to a hydrophobic pocket defined by the connecting loop $\beta 1-\alpha 1$ from the N-terminal domain (residues Val13-Gly16), the ends of strands $\beta 8$ and $\beta 9$, and the connecting loop $\beta 10-\alpha 9$ from the C-terminal domain. The ribose oxygens $\mathrm{O}^{\prime}$ ' and $\mathrm{O}^{\prime}$ ' interact with the carboxylate group of Glu282, a conserved glutamate residue of the $\mathrm{EX}_{7} \mathrm{E}$ motif, present in some members of the GT-B-fold superfamily $(12,30)$. Interestingly, the $\alpha-\mathrm{PO}_{4}$ of GMP displays a different conformation when compared to the position observed in the PimA • GDP and PimA •GDP-Man crystal structures (Figure $2 \mathrm{C}$; 18). The $\alpha-\mathrm{PO}_{4} \mathrm{O} 1$ ' makes new hydrogen bond with the main chain of Gly16, almost reaching the position of the $\beta-\mathrm{PO}_{4} \mathrm{O}^{\prime}$ ' in GDP and GDP-Man (Figure 2C). The catalytic loop (residues 268 to 277) adopts a different structural arrangement closer to the $\alpha-\mathrm{PO}_{4}$ (r.m.s.d. of $0.9 \AA$ and $1.0 \AA$ for 10 equivalent residues in PimA•GDP and PimA•GDP-Man, respectively). 

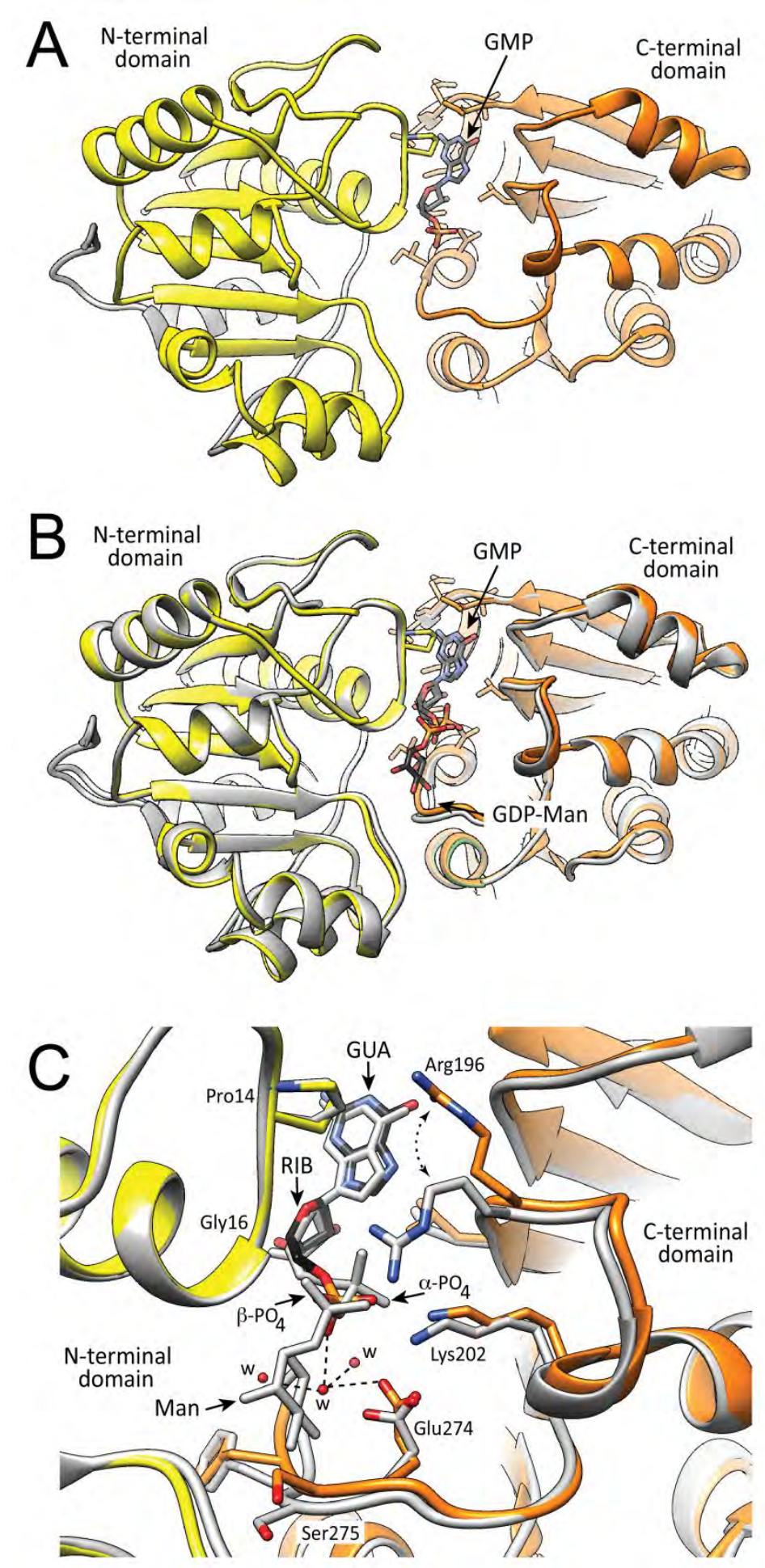

Figure 2. The crystal structure of PimA in complex with GMP. A. Overall structure of $M s$ PimA in complex with GMP. The N-terminal and C-terminal domains are shown in yellow and orange respectively. B. Structural superposition between $M s$ PimA $\bullet$ GMP and $M s$ PimA $\bullet$ GDP-Man (grey) complexes. $C$. Structural superposition of GDP-Man and GMP as observed in the crystal structures of the corresponding crystal structures. 
The main-chain oxygen of Ser275 makes a hydrogen bond with the structural water molecule W1, which in turn interacts with the $\alpha-\mathrm{PO}_{4} \mathrm{O} 3$ ', and the side-chain of Glu274 (11). The $\varepsilon$ amino group of the essential Lys202 lateral chain, which in the GDP and GDP-Man complexes established an important polar interaction with the $\beta-\mathrm{PO}_{4} \mathrm{O} 2$ ', makes now a hydrogen bond with the $\alpha-\mathrm{PO}_{4} \mathrm{O} 2$ '. In contrast, the lateral chain of another essential residue Arg196, also involved in the interaction with the $\beta-\mathrm{PO}_{4}$, moves away from the active site (Figure 2C). Thus, the conformational changes observed in the active site of the $M s$ PimA-GMP complex raise the question about the precise structural elements of the nucleotide sugar donor involved in the conformational transitions observed in PimA.

\section{Chemical synthesis of GDP-Man derivatives}

We dissected the GDP-Man structure generating truncated versions as an approach to determine, what are the structural component/s within the native donor substrate structure responsible for the (i) fold-switch from inactive extended to active extended, (ii) motion from extended active to compact active and (iii) stabilization of the active compact conformation. Thus, we selected a series of GDP-Man derivatives: those containing the nucleobase ring, including GDP, GMP, guanosine (GNO) and guanine (GUA); and those containing the mannose ring, mannose-phosphate (Man-P) and mannose-pyrophosphate-ribose (Man-PPRIB). In addition, we have synthetized pyrophosphate-ribose (PP-RIB), which connects to the mannose residue through the $\beta$-phosphate and to the nucleobase through the ribose ring (Figure 3A).

The synthetic derivatives Man-PP-RIB and PP-RIB were designed as $\beta$ - $O$-methyl glycosides at the ribose moiety to ensure a well-defined $\beta$-anomeric configuration, mimicking the electronic and conformational properties of the natural substrate and minimizing any undesired side-interactions and steric hindrance. Two different synthetic approaches were 
used for the preparation of the syntetic derivatives. In the case of Man-PP-RIB the key reaction involved the phosphate-phosphate coupling between the activated forms of Man-P and P-RIB (see the chemical synthesis of Man-PP-RIB, in Scheme S1 of the Supplementary Information). Commercially available D-ribose was converted into protected compound 1 that, after removal of the trityl group, furnished 2 in good yield. The free hydroxyl at C-5 was phosphorylated using dibenzyl $N, N$-diisopropylphosphoramidite and the resulting phosphite ester was oxidized in situ with ${ }^{\mathrm{t}} \mathrm{BuOOH}$ giving compound $\mathbf{3}$ that was deprotected and activated as imidazolyl phosphate yielding 4. Finally, phosphate coupling of activated compound 4 with $\alpha$-D-mannose-1-phosphate using $\mathrm{MgCl}_{2}$ afforded Man-PP-RIB in good overall yield. PP-RIB was prepared through a sequential phosphorylation reaction using the phosphoramidite/oxidation method (see the Chemical synthesis of PP-RIB in Scheme S2 of the Supplementary Information). Commercially available D-ribose was converted into protected compound $\mathbf{5}$ and subsequently, the free hydroxyle at C-5 was phosphorylated using the phosphoramidite/tBuOOH technique furnishing compound $\mathbf{6}$ which was debenzylated though Pd-catalyzed hydrogenation to give 7. Treatment of 7 with $N, N$ diisopropylphosphoramidite / $\mathrm{BuOOH}$ to give $\mathbf{8}$, followed by removal of the isoproplidene group and debenzylation through hydrogenolisis furnished PP-RIB in good overall yield.

\section{The role of the $\mathrm{\beta PO}_{4}$ in the generation of the active compact conformation of MsPimA}

First, we determined the impact of the nucleobase derivatives interaction on the conformation of $M s$ PimA by limited proteolysis. We have previously demonstrated that in the presence of GDP, which comprises the nucleobase, the ribose moiety and the $\alpha-\mathrm{PO}_{4}$ and $\beta-\mathrm{PO}_{4}, M s \mathrm{PimA}$ is barely cleaved, even after 90 min incubation with elastase (18). 

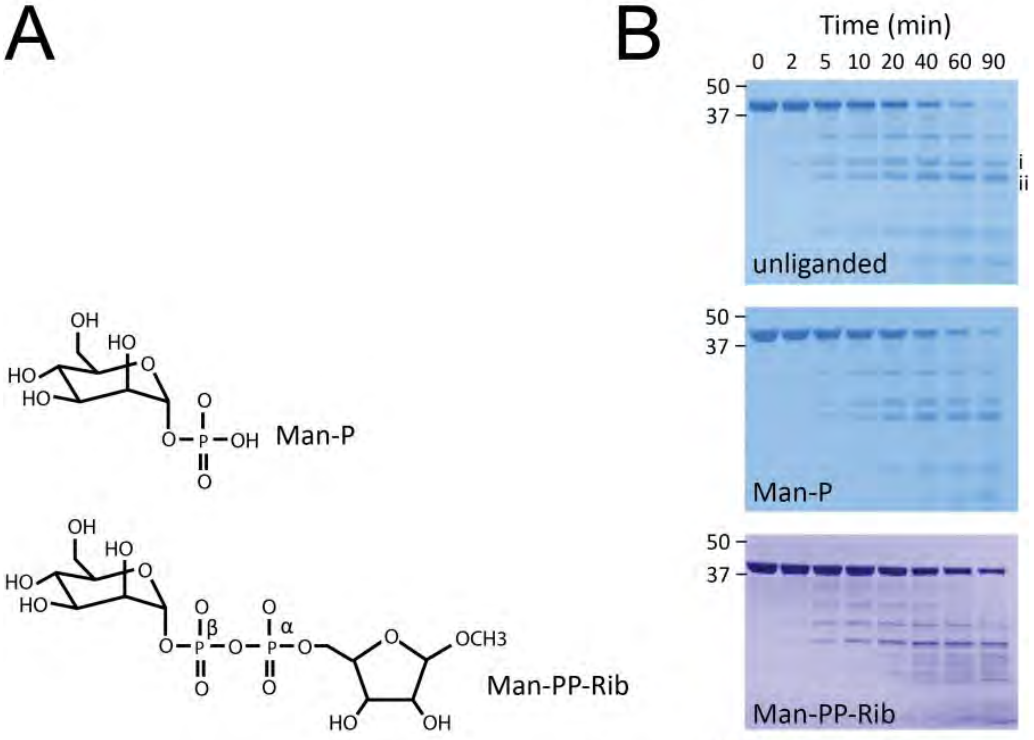

$$
\text { II }
$$
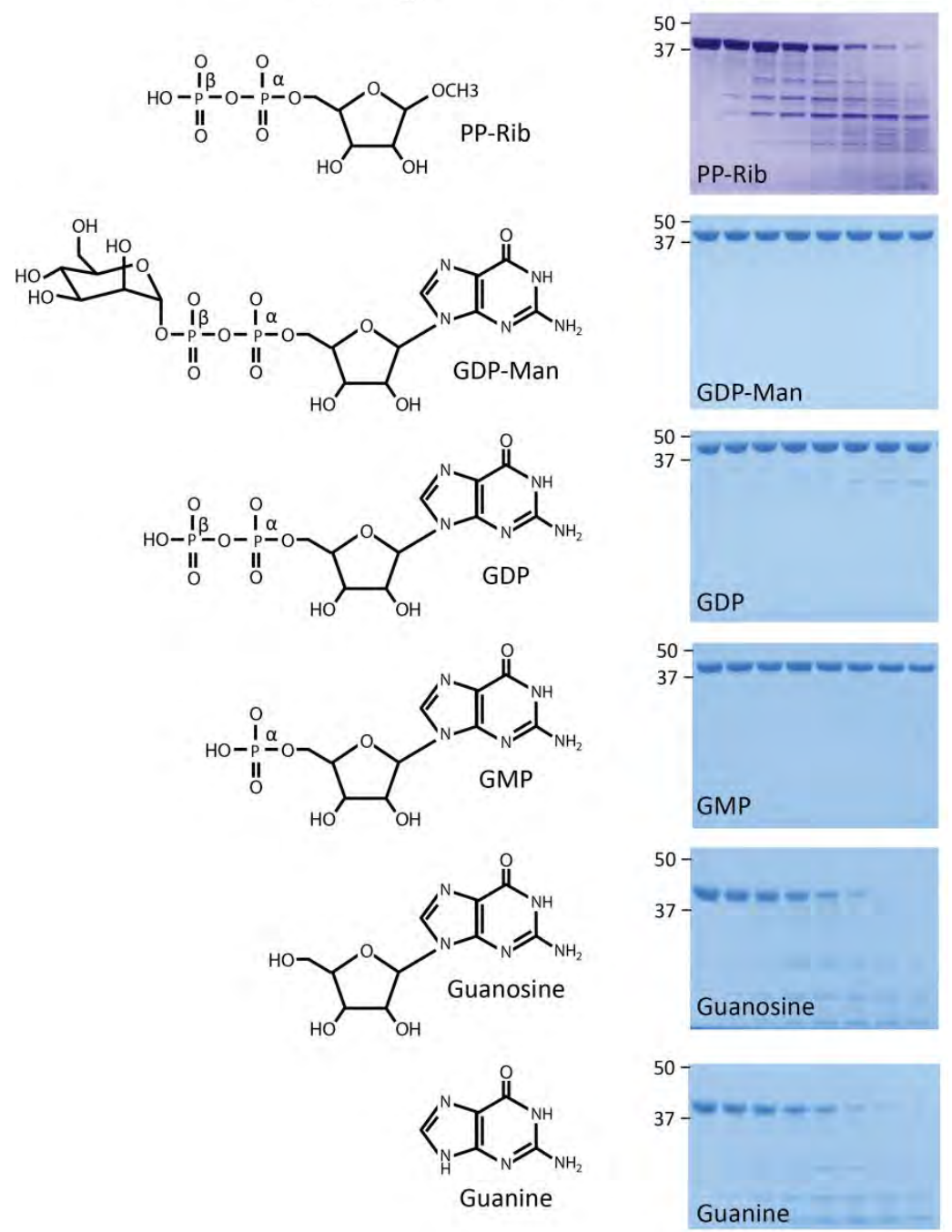

Figure 3. Endoproteolytic cleavage of $M s \operatorname{PimA}$ in the presence of GDP-Man derivatives. $A$. Chemical structure of GDP-Man derivatives. $B$. SDS-PAGE showing the elastase cleavage profile for MsPimA preincubated with GDP-Man derivatives. 
Here we see, as depicted in Figure 3B, that when MsPimA was incubated with GMP, in which only the $\alpha-\mathrm{PO}_{4}$ of GDP is present, the enzyme was mostly protected from the degradation by the protease. GNO, lacking the pyrophosphate group of GDP, only partially protected $M s$ PimA from the action of the elastase. In contrast, $M s$ PimA was rapidly degraded after incubation with the nucleobase GUA, and PP-RIB showing a similar proteolytic profile to that observed with the unliganded form of the enzyme (Figure 3B). It is worth noting that we have previously microsequenced the two predominant small species of 23 and $15 \mathrm{kDa}$ revealing the sequences (i) SAMRS, located in $\alpha 9$ and (ii) SFADA, in the connecting loop $\beta 7-\beta 8$ at the junction between $\mathrm{N}$ - and C-terminal domains. Interestingly, the $\alpha 9$ helix contains two critical residues involved in donor substrate recognition: Asp253, which interacts with N2 of the guanidyl group of GDP-Man, conferring $M s$ PimA its specificity for the nucleoside; and Lys256, which participates in ribose binding (11, 18; Figure 3). Secondly, we studied by nearUV circular dichroism (CD) the tertiary structural changes of PimA induced by the nucleotide derivatives. The near-UV CD spectrum of $M s \mathrm{PimA}$ in its unliganded form in solution exhibited a broad positive signal characteristic of aromatic side chains, namely phenylalanines, tyrosines and tryptophans, localized in the protein (Figure 2B). The addition of GDP induced a gain of the tertiary structure constrains on aromatic residues, as indicated by an increment of the dichroic signal (Figure 4A). The addition of GMP also increased the dichroic signal on MsPimA, however not as high as that observed for the PimA $\bullet$ GDP complex. The incubation of MsPimA with GNO generated small, but still detectable differences in the near-UV CD profile. In contrast, the addition of the nucleobase GUA or that of PP-RIB, did not change the spectrum. 

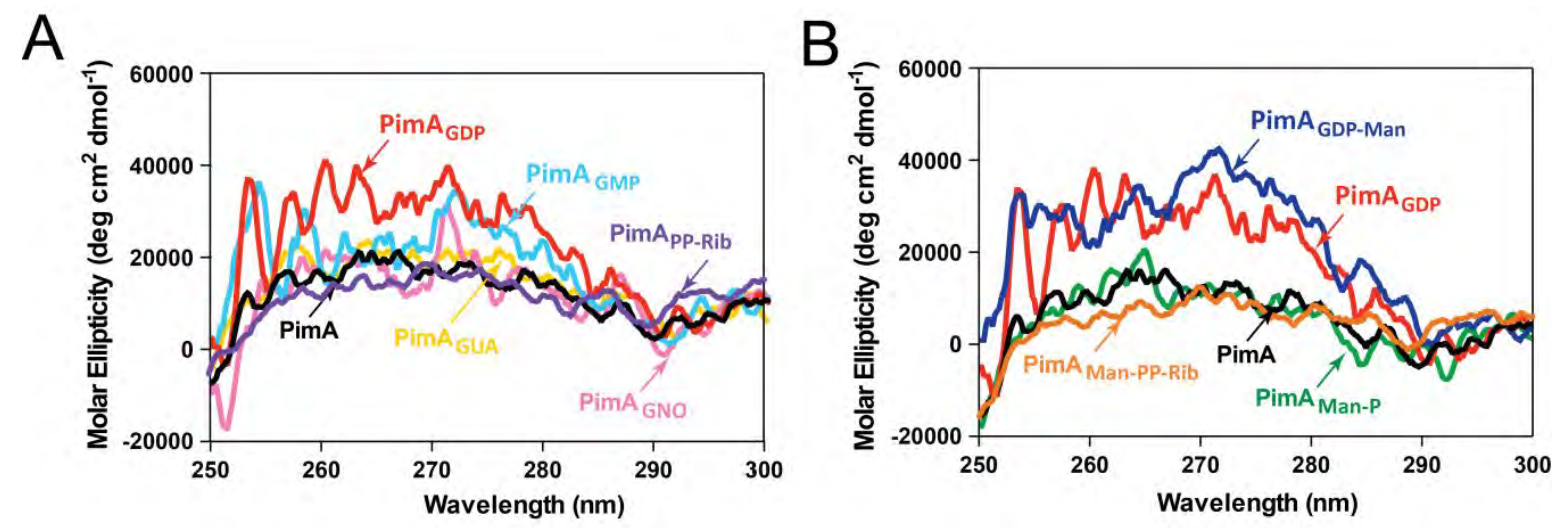

Figure 4. Near CD of MsPimA in the presence of GDP-Man derivatives. $A$. Near-UV CD spectra for the unliganded form of $M s$ PimA (black) and the $M s \mathrm{PimA} \bullet \mathrm{GDP}$ (red), $M s$ PimA $\bullet \mathrm{GMP}$ (light blue), $M s \mathrm{PimA} \cdot \mathrm{GNO}$ (pink), $M s \mathrm{PimA} \cdot \mathrm{PP}-\mathrm{Rib}$ (purple) and $M s \mathrm{PimA} \cdot \mathrm{GUA}$ (yellow) complexes. $B$. Near-UV CD spectra for the unliganded form of $M s$ PimA (black) and the $M s$ PimA $\cdot G D P-M a n$ (blue), $M s \mathrm{PimA} \bullet \mathrm{Man}-\mathrm{PP}-\mathrm{Rib}$ (orange) and $M s \mathrm{PimA} \bullet \mathrm{Man}-\mathrm{P}$ (green) complexes.

Thermal unfolding followed by $\mathrm{CD}$ at a wavelength of $222 \mathrm{~nm}$ showed similar apparent melting temperatures $\left(T_{\mathrm{M}}\right)$ of $37.0,36.6,36.4,36.4$ and $36.2{ }^{\circ} \mathrm{C}$, for the $M s \mathrm{PimA} \cdot \mathrm{GMP}$, $M s$ PimA $\bullet$ GNO, $M s$ PimA $\bullet$ GUA, $M s$ PimA $\bullet P P-R I B$ and the unliganded form of $M s$ PimA, respectively (Table 1). An important observation was that the $T_{\mathrm{M}}$ of $M s \mathrm{PimA} \cdot \mathrm{GDP}$ complex was $46.4{ }^{\circ} \mathrm{C}$, indicating that this state is $c a .10{ }^{\circ} \mathrm{C}$ more stable than $M s$ PimA or the $M s$ PimA $\bullet G M P, M s$ PimA $\bullet G N O, M s$ PimA $\bullet G U A$ complexes. PimA alone displayed a van't Hoff enthalpy change $(\Delta \mathrm{H})$ of about $70 \mathrm{kcal} / \mathrm{mol}$. The addition of GMP, GNO, GUA or PPRIB had no effect on $M s$ PimA $\Delta \mathrm{H}$ parameters. The addition of GDP significantly increased the $\Delta \mathrm{H}$ to $155 \mathrm{kcal} / \mathrm{mol}$. The $K_{d}$ determination by ITC revealed that GDP-Man and GDP bind to $M s$ PimA in enthalpy-driven reactions with dissociation constants, $K_{d}$ of $0.23 \mu \mathrm{M}$ and 0.03 $\mu \mathrm{M}$, respectively (11). In contrast, no binding of GMP, GNO, GUA could be detected to $\operatorname{MsPimA}\left(K_{d}>100 \mu \mathrm{M}\right)$ under the same experimental conditions (see Experimental Procedures section). Therefore, there is a clear/direct correlation between the chemical complexity of the derivative and the gain of ternary structure consistent with their binding 
interactions in $M s$ PimA. Only the GDP derivative binding, which contains the $\beta-\mathrm{PO}_{4}$, triggered the stabilization of $M s$ PimA (Table 1).

Table 1. Unfolding parameters of free and MsPimA bound to GDP-Man derivatives, measured by CD.

\begin{tabular}{|c|c|c|}
\hline & $\mathbf{T m}(\mathbf{-} \mathbf{C})$ & $\Delta \mathbf{H}(\mathbf{k c a l} / \mathbf{m o l})$ \\
\hline Unliganded & 36.3 & 67.3 \\
\hline GDP-Man & 45.5 & 165.7 \\
\hline GDP & 47.5 & 155.6 \\
\hline GMP & 37.0 & 70.1 \\
\hline GNO & 36.6 & 63.4 \\
\hline GUA & 36.4 & 66.9 \\
\hline Man-P & 36.5 & 59.0 \\
\hline Man-PP-Rib & 36.2 & 66.3 \\
\hline PP-Rib & 36.4 & 64.5 \\
\hline
\end{tabular}

The mannose residue is dispensable for the generation of the active compact conformation of MsPimA

How does the sugar ring binding impact the conformation of $M s$ PimA? The crystal structure of the $M s$ PimA $\bullet$ GDP-Man complex revealed that the mannosyl moiety of GDP-Man is stabilized within the active site cleft through several hydrogen bonding contacts mainly located in the catalytic loop: O3 makes a hydrogen bond with the main-chain of Ser275; the O4 makes important hydrogen bonding interactions with the lateral chain of Glu274 and the main chain of Phe276; whereas the O6 engages the nitrogen atom of the main chain of Ile278 (11). As depicted in Figure 3B, while $M s$ PimA was completely protected from proteolytic digestion by GDP-Man, as also observed for GDP, the enzyme was not protected from elastase degradation after incubation with Man-P or Man-PP-RIB, where the $\beta-\mathrm{PO}_{4}$ is present. The near-UV CD spectra of the $M s$ PimA $\bullet G D P-M a n$ complex revealed clear differences in the dichroic signal with respect to the unliganded form of $M s$ PimA reflecting variations in the ternary structure of the enzyme. Interestingly, the addition of Man-P or Man-PP-RIB, did not change the spectrum when compared to that of the unliganded form of MsPimA (Figure 4B). The thermal unfolding profile of $M s \mathrm{PimA} \cdot \mathrm{GDP}-\mathrm{Man}, M s \mathrm{PimA} \bullet \mathrm{Man}-\mathrm{P}$ and $M s \mathrm{PimA} \bullet \mathrm{Man}-$ 
PP-RIB showed remarkable different $T_{\mathrm{M}}$ values of $44.3,36.5^{\circ} \mathrm{C}$ and $36.2{ }^{\circ} \mathrm{C}$ (Table 1). The addition of GDP-Man significantly increased the $\Delta \mathrm{H}$ to $165 \mathrm{kcal} / \mathrm{mol}$, whereas the addition of Man-P or Man-PP-RIB had no effect on $M s$ PimA $\Delta \mathrm{H}$ parameters (Table 1).

\section{The 'active extended' to 'active compact' transition of MSPimA as visualized by SAXS}

Small angle X-ray scattering (SAXS) has proved to be a powerful technique capable of providing structural information of flexible and dynamic proteins in solution $(15,19)$. The interatomic distance distribution function $(P(r))$ computed for $M s$ PimA $\bullet$ GDP-Man, $M s$ PimA $\bullet \mathrm{GMP}, M s$ PimA $\bullet P P-R I B, M s P i m A \bullet M a n-P$, and $M s$ PimA $\bullet$ Man-PP-RIB is shown in Figure 5A. (see also Table S2). The molecular mass determined from the scattering data confirmed that the protein is monomeric in solution in agreement with size exclusion chromatography and analytical ultracentrifugation $(11,18)$. The radius of gyration $\left(R_{\mathrm{g}}\right)$ value obtained for $M s$ PimA $\cdot G D P-M a n(29.3(5) \AA)$ and the unliganded form (30.8(4) $\AA$ ), revealed a reduction in $R g\left(\Delta R_{g}\right)$ of $-1.5 \AA$, indicating that the complex is in a compact conformation with respect to $M s \mathrm{PimA}$ alone, a similar behavior observed for the $M s \mathrm{PimA} \cdot \mathrm{GDP}$ complex (29.2(4) $\AA$; $\left(\Delta R_{g}\right.$ of $\left.-1.6 \AA\right)$. Interestingly, the addition of GMP $\left(R_{g}=29.6(3) \AA\right)$ also trigerred the formation of a compact conformation as visualized in the crystal structure of the $M s$ PimA $\cdot G M P$ complex (Figure 2). In contrast, the addition of PP-RIB $\left(R_{g}=31.1(4) \AA\right)$, Man-P $\left(R_{g}=31.0(5) \AA\right)$ or Man-PP-RIB $\left(R_{g}=31.2(4) \AA\right)$ to $M s$ PimA showed similar $R_{\mathrm{g}}$ values as for the unliganded form of the enzyme $\left(R_{g}=30.8(4) \AA ; 21\right)$. Furthermore, the normalized Kratky plot (Figure 5B) indicates that $M s$ PimA in complex with GDP-Man, GDP or GMP is more symmetric (closer to following Guinier's approximation), than MsPimA unliganded or in complex with PP-RIB, Man-P or Man-PP-RIB. Normalized $P(\mathrm{r})$ function distributions are shown in Figure 5C. 

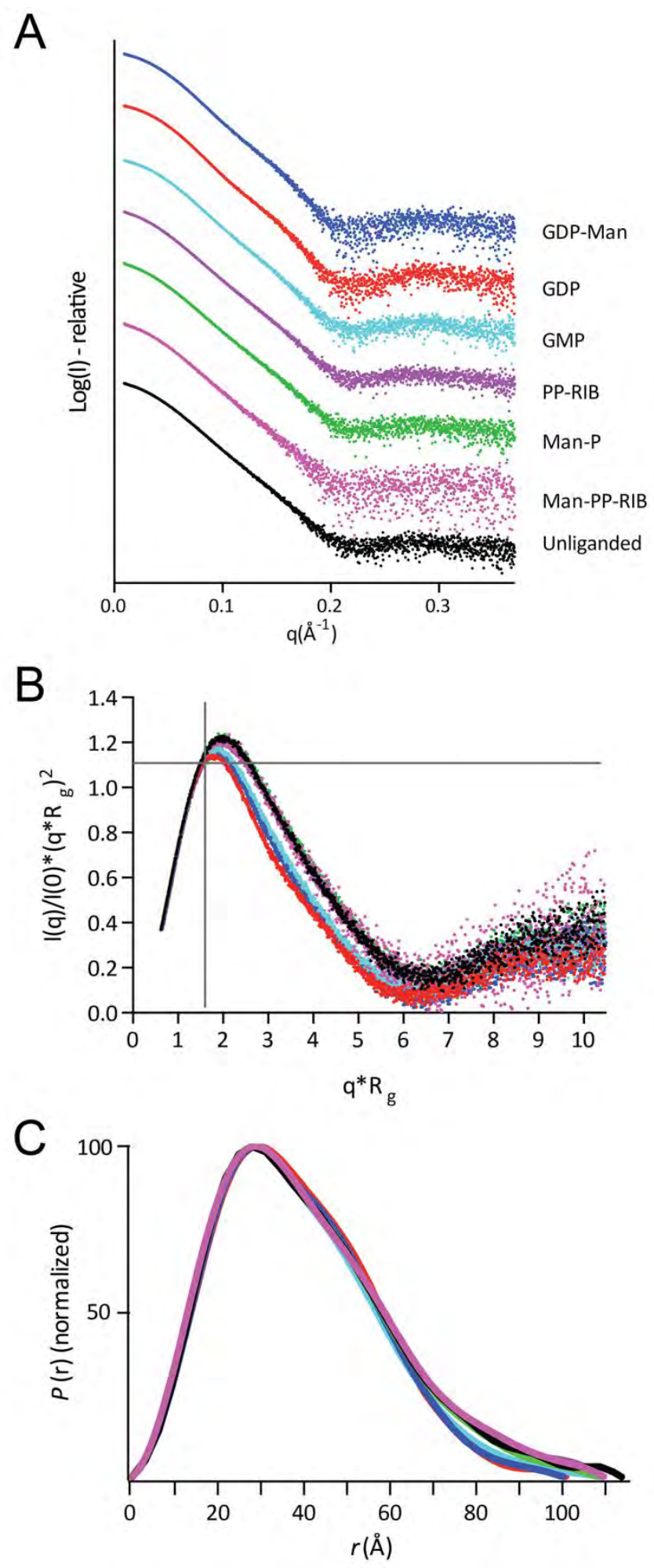

Figure 5. The active extended to active compact transition as visualized by SAXS. A. Scattering curves of unliganded $M s$ PimA (black), MsPimA $\bullet$ GDP-Man (blue), $M s$ PimA $\bullet$ GDP (red), $M s$ PimA $\bullet$ GMP (light blue), MsPimA $\bullet$ Man-PP-Rib (pink), MsPimA $\bullet P P-R i b ~(v i o l e t)$ and PimA $\bullet$ Man-P (green) complexes. B. Normalized Kratky plot of unliganded $M s$ PimA (black), MsPimA $\cdot$ GDP-Man (blue), $M s$ PimA $\bullet \mathrm{GDP}$ (red), $M s$ PimA $\bullet \mathrm{GMP}$ (light blue), $M s$ PimA $\bullet$ Man-PP-Rib (pink), MsPimA $\bullet P P-$ Rib (violet) and PimA $\bullet$ Man-P (green) complexes. Lines crossing at $q * R_{g}=\sqrt{3}$ with a magnitude of 1.104 indicates the expected maxima of a globlular particle obeying Guinier's approximation. $C$. Normalized $P(\mathrm{r})$ function distributions. 


\section{DISCUSSION}

The crystal structures of the $M s$ PimA $\bullet$ GDP-Man and $M s$ PimA $\bullet$ GDP complexes were obtained in an 'active compact' conformation (Figure 1) (11). Interestingly, the $M s \mathrm{PimA} \cdot \mathrm{GMP}$ complex also revealed an active compact conformation, but with important differences on the structural arrangement of the GMP molecule (Figure 2) in the active site when compared with $M s$ PimA $\bullet G D P-M a n$ and $M s$ PimA $• G D P$ complexes (Figure 1). This reorganization changes the previously seen interactions with the $\beta-\mathrm{PO}_{4}$ substantially and instead reveals new ones with the $\alpha-\mathrm{PO}_{4}$. From a catalytic point of view, the reaction catalyzed by PimA involves the cleavage of the phosphor sugar bond. Bearing this in mind, the different $\alpha-\mathrm{PO}_{4}$ disposition within the active site can be seen as an attempt to accommodate the GMP molecule and to stabilize temporary and weakly key interactions originally needed for the $\beta-\mathrm{PO}_{4}$ cleavage. Actually, SAXS analysis of the corresponding $M s$ PimA $\bullet G D P-M a n, M s P i m A \bullet G D P$ and $M s$ PimA $\cdot$ GMP complexes are in agreement with the ability of GDP-Man, GDP and GMP to stabilize the 'active compact' conformation (Figure 5). It is also observed by limited proteolysis, where $M s \mathrm{PimA}$ is less prone to protease cleavage when bound to GDP-Man, GDP and GMP (Figure 3), and by a substrate-complexity proportional gain in tertiary structure by near-UV spectra (Figure 4). Nevertheless, this gain is not accompanied by an increase in the van't Hoff enthalpy suggesting no stabilization. Experimentally calculated $T_{\mathrm{M}}$ from our CD assays are in agreement with this observation, since only GDP-Man and GDP markedly stabilized $M s$ PimA by $c a \cdot 10^{\circ} \mathrm{C}$ and the derivatives including GMP, GNO, GUA did not (Figure 4). The stabilization and binding are supported by the $K_{d}$ parameters determined by ITC which revealed that GDP-Man and GDP bind to MsPimA in enthalpydriven reactions with dissociation constants, $K_{d}$ in the micromolar range (11) whereas no binding of GMP, GNO, GUA could be detected $\left(K_{d}>100 \mu \mathrm{M}\right)$ (Figure 6). 
The GMP structural data - crystal structure and SAXS, limited proteolysis and Near-UV spectra serve as evidence to propose that the nucleotide moiety linked at least to the $\alpha-\mathrm{PO}_{4}$ is able to weakly bind and temporarily stabilize the active conformation but, apparently it does not provide the sufficient energy to form a high affinity binding. Despite only a weak binding/stabilization is observed, the effect is still detectable by SAXS, limited proteolysis, and near-UV CD spectra. The GMP-bound form would also reflect the expected rigidity needed for the active sites in order to stabilize ligand-enzyme interactions $(7,8)$. Therefore, we propose the 'nucleoside moiety $+\alpha-\mathrm{PO}_{4}+\beta-\mathrm{PO}_{4}$ ' as minimal structure required to stabilize this conformation. Hence, the GMP effect on PimA might be seen as a sort of artifact due to the structural analogy with the native substrate. The essential interactions directly involved in the binding to $\beta-\mathrm{PO}_{4}$, mainly arising from Gly16, Arg196, and Lys202, are distorted when GMP accommodates within the active site (Figure 2). In addition, the disposition of the catalytic loop containing Ser275 and Glu274 is also altered. As our biochemical data showed, it is reasonable to propose that these residues and their interaction with the $\beta-\mathrm{PO}_{4}$ confer the specificity for the high affinity binding and the stabilization energy to achieve a competent active site in the active compact conformation. Additionally, we also studied the contribution of the mannose moiety and its derivatives. SAXS data for $M s \mathrm{PimA} \bullet \mathrm{Man}-\mathrm{P}, M s \mathrm{PimA} \bullet \mathrm{Man}-\mathrm{PP}$ and $M s \mathrm{PimA} \bullet \mathrm{PP}-\mathrm{RIB}$, complexes also displayed similar behavior to the unliganded form. Our CD, limited proteolysis and ITC experiments performed in the presence of the above mentioned truncated versions are in agreement with our hypothesis showing no thermal stabilization in the presence of PP-RIB, Man-PP-RIB, and Man-P nor binding detected to $M s \operatorname{PimA}\left(K_{d}>100 \mu \mathrm{M}\right)$ (Figure 6) and in consequence, they were more prone to the protease's activity (Figure 3). In conclusion, only GDP-Man and GDP exhibit high affinity binding constants and the highest stabilization energies to interact with the active compact state. Therefore, the simultaneous presence of the nucleotide moiety and 
the $\beta-\mathrm{PO}_{4}$ moieties is indispensable to (i) provide the high affinity binding and (ii) to generate a competent active site through the active compact conformation stabilization. Nevertheless, the nucleotide moiety bound to at least the $\alpha-\mathrm{PO}_{4}$ is able to interact weakly with PimA favoring the active compact conformation state due to a delocalization of the $\alpha-\mathrm{PO}_{4}$, almost reaching the $\beta-\mathrm{PO}_{4}$ location, and establishing a mimicking interaction.
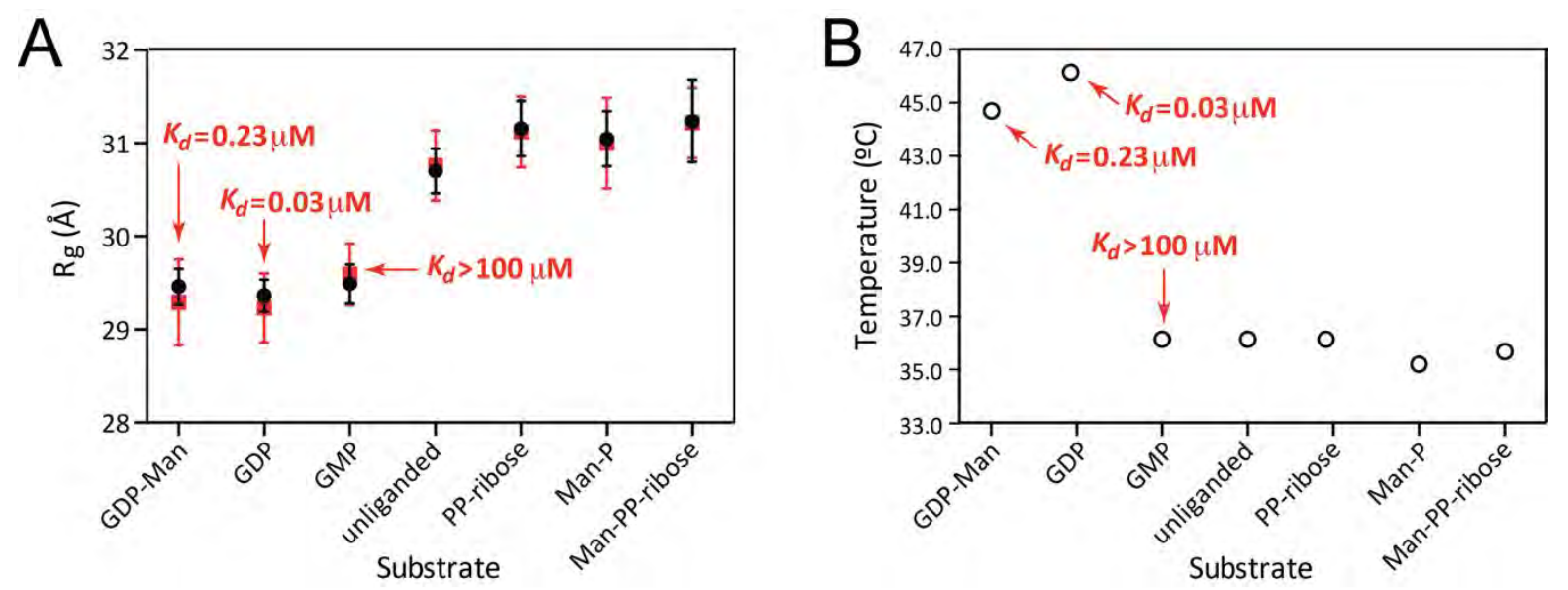

Figure 6. Graph showing the $R_{g}, K_{d}$ and $T_{M}$ values obtained for PimA in the presence or absence of different GDP-Man derivatives. $A$. $R g$ Guinier (filled black circles) and $R g$ real space (filled red squares) values of unliganded $M s$ PimA, $M s$ PimA $\bullet$ GDP-Man, $M s$ PimA $\bullet$ GDP, $M s$ PimA $\bullet$ GMP, $M s \mathrm{PimA} \bullet \mathrm{GNO}, \quad M s \mathrm{PimA} \bullet \mathrm{GUA}, \quad M s \mathrm{PimA} \bullet \mathrm{Man}-\mathrm{PP}-\mathrm{Rib}, \quad M s \mathrm{PimA} \bullet \mathrm{PP}-\mathrm{Rib}$ and $M s \mathrm{PimA} \bullet$ Man-P complexes are ploted with reference to the vertical left- and right-axis, respectively. The $K_{d}$ values are indicated next to the ploted $R g$ values. $B . T_{M}$ (empty circles) values of unliganded $M s$ PimA, $M s \mathrm{PimA} \bullet \mathrm{GDP}-\mathrm{Man}, M s \mathrm{PimA} \bullet \mathrm{GDP}, M s \mathrm{PimA} \bullet \mathrm{GMP}, M s \mathrm{PimA} \bullet \mathrm{GNO}, M s \mathrm{PimA} \bullet \mathrm{GUA}, M s \mathrm{PimA} \bullet$ ManPP-Rib, $M s$ PimA $\bullet P P-R i b$ and $M s$ PimA $\bullet$ Man-P complexes are ploted with reference to the vertical leftand right-axis, respectively. The $K_{d}$ values are indicated next to the ploted $T_{M}$ values.

Two catalytic mechanisms have been proposed for retaining GTs, (i) a double displacement mechanism that involves a nucleophilic residue that forms a covalent sugarenzyme intermediate, and in the absence of a nucleophilic residue, (ii) a substrate-assisted front-face reaction mechanism that involves the formation of an oxocarbenium ion-like transition state $(14,31,32,33)$. In support of the substrate-assisted front-face reaction mechanism, recent structural work on GT-A GTs using a quick-soaking method has provided unprecedented insights by trapping snapshots along the reaction cycle $(33,34,35)$. That is the 
case for the retaining GTs glucosyl-3-phosphoglycerate synthase (GpgS) and $\alpha-1,3-$ galactosyltransferase $(\alpha 3 \mathrm{GalT})$. Although the latter contains a putative nucleophile in the active site (Glu317), the native ternary complexes determined in a productive mode for catalysis in the presence of their sugar donor, acceptor substrates, and metal cofactors, support the substrate-assisted front-face reaction mechanism, suggesting a common reaction mechanism for all retaining GTs $(34,36,37)$.

PimA and PimB are two GT-B GT that exhibit a putative nucleophile in the active site (residues Glu274 and Glu290, respectively). But, although the mutation Glu274Ala in PimA completely abolished the enzyme's activity (18), the Glu290Ala mutation in PimB did not reduce activity to the same extent (38), suggesting that this conserved glutamate residue might be important for sugar binding and stabilization of the transition state. Thus, it has been proposed that PimA is likely to follow a substrate assisted front-face reaction mechanism (39). When the position of the NDP-sugar donor within the active site was compared with closest homologs WaaG and MurG in the presence of UDP-2-deoxy-2-fluoroglucose (UDP2FGlc), the pyrophosphate-sugar moiety in the PimA complex has a different conformation compared to WaaG and MurG. Nevertheless, GDP-Man is found in a 'bend back' conformation relative to the pyrophosphate group and sugar residues which is conserved in all retaining GTs, exposing the anomeric C1 carbon to nucleophilic attack (40). Unfortunately, there is no structural information of PimA in the presence of the acceptor PI, but QM/MM calculations for two of the closest structural and functional homologs, trehalose-6-phosphate synthase (OtsA) and MshA, support a substrate-assisted front-face reaction mechanism (31, 41).

OtsA is an extensively studied retaining GT-B that uses UDP-Glucose as donor and Glc-6P as acceptor to form trehalose-6P. The crystal structure of OtsA in complex with the glycomimetic inhibitor validoxylamine A 6'-O-phosphate and UDP traped a putative 
transition state-like arrangement in the active site (42). This structure provided structural evidences for a front-face reaction mechanism, which was further supported by kinetic isotope effects that indicated the existence of a highly dissociative oxocarbenium ion-like transition state (31). These experimental obsrvations are in agreement with QM/MM calculations, which suggest that the reaction is likely to happen stepwise and that it involves the formation a short-lived oxocarbenium-ion-like intermediate. These calculations suggested the phosphate group acts as a base enhancing the nucleophilicity of the incoming acceptor molecule (32).

Another example, the GT4 MshA from Corynebacterium glutamicum involved in the mycothiol biosynthesis, illustrates a very similar scenario to that possibly undergone by PimA. MshA is a metal-independent GT-B which uses 1-L-myo-inositol-1-phosphate (m-Ins1P), and UDP-GlcNAc as activated donor, transferring the GlcNAc to the $3^{\text {rd }}$ position of the inositol ring (40). In this case, a concerted substrate-assisted front-face reaction mechanism has been proposed where the m-Ins-1P O3 performs a nucleophilic attack on the anomeric $\mathrm{C} 1$ carbon in GlcNAc. As opposed to the stepwise front-face reaction mechanism of OtsA, here the m-Ins-1P O3 is concomitantly deprotonated by the oxygen 3 of the $\beta-\mathrm{PO}_{4}(\mathrm{O} 3 \mathrm{~B})$ group that acts as leaving group. The key process leading to the transition state clearly corresponds to the breaking of the GlcNAc $-\beta-\mathrm{PO}_{4}$ bond with the concomitant strengthening of the hydrogen bond between the $\beta-\mathrm{PO}_{4} 3 \mathrm{~B}$ and the $\mathrm{HO} 3$ of the m-Ins-P (41). A close inspection of the interaction between the MshA residues and the $\beta-\mathrm{PO}_{4}$ reveals that $\mathrm{MshA}$, similarly to PimA, has a glycine, Gly23, (equivalent to Gly16 in PimA) coordinating the pyrophosphate. The Gly16, which belongs to the named GlyGly loop - $\beta 1 / \alpha 1$ loop located at the N-terminal domain - is conserved in other GT-B superfamily members and its motion appears to be essential for catalysis in the closest structural homologs (37, 43). In addition, Arg231 in MshA (equivalent Arg196 in PimA) is stabilizing and placing $\beta-\mathrm{PO}_{4}$ in close contact with the 
O3 nucleophile from the donor. The mutations of Arg196Ala in PimA completely abolished the activity (11) indicating the relevance of this residue for catalysis. Our structural results, the comparison with retaining GT-B homologs and computational studies support a front-face reaction mechanism that involves the formation of an oxocarbenium ion-like transition state, where the $\beta-\mathrm{PO}_{4}$ must be placed spatially close to the acceptor group in order to assist in catalysis. The residues responsible of the proper cleavage and spatial location of $\beta-\mathrm{PO}_{4}$ are crucial for catalysis corroborating observations that the $\beta-\mathrm{PO}_{4}$ is essential. Nonetheless, further structural and computational evidence are required for PimA.

It is well established that for the GT-B superfamily members the acceptor binding site is located at the $\mathrm{N}$-terminal domain whereas the donor substrate binds mainly to the $\mathrm{C}$-terminal domain. The catalytic cleft is formed between the two spatially separated domains upon an 'open-to-closed' conformational change. This domain rearrangement has been extensively characterized for PimA and predicted and/or observed for some other members of the GT-B superfamily $(14,46,47,48)$. PimA is therefore an outstanding example of GT-B flexibility and intrinsic dynamic properties for the study of GT-B enzymes. The coexistence of different substates in solution serves as evidence to show how PimA samples, at least, three different conformations. These motions are mainly happening at the $\mathrm{N}$-terminal domain of the protein unveiling the potential relevance of the acceptor substrate impact on conformational changes. Since the range of acceptors is wider than the range of donors, in terms of chemical structure complexity, this domain exhibits a greater diversity and flexibility of secondary structure elements arrangements $(37,49)$ which in turn, facilitates the adaptability to interact with acceptor substrates. However, despite the apparent structural disparity in the N-terminal domain for some GT-B members, evolution has driven the GT-B function based on (i) conserved motifs responsible for the recognition and binding to the substrates and (ii) high intrinsic flexibility presenting a shared trend in dynamics - 'open to closed' motion. The 
open-to-closed movement, as a common feature, indicates that it is efficient and functional motion required to perform the catalysis as it places donor and acceptor substrates in close spatial contact $(37,43)$.

For PimA, the scenario seems to be even more complex due to a fold switch prior to the 'open-to-closed' motion. The fold switch is considered the rate-limiting step of the glycosylation reaction and it has been defined as the activation mechanism of PimA (15). Once the fold switch occurs, it is expected that PimA populates an active extended conformation, not-yet structurally characterized. This domain 'open-to-closed' conformational change allows critical residues from the $\mathrm{N}$ - and $\mathrm{C}$-terminal domains to bring together, and build a functionally competent active site (Figure 1). Up to the date, it was observed that the donor substrate presence induced the 'open-to-closed' motion in some members $(13,37)$. However, the dynamic process and the structural determinants responsible for it remained unresolved. As we have shown, the residues intervening in the $\beta-\mathrm{PO}_{4}$ cleavage are critical to stabilizing this state and to the catalysis. Indeed, our results are in agreement with the evidence for a correlation between an increase in the structural complexity of the substrate and the derived conformational changes since they are stabilized by the increasing number of key side chain interactions while the substrate binding provides the energy to form the transition state (11). Conversely, a decrease in the donor substrate structure complexity would lead to a lower binding and stabilization interactions. For PimA, this agrees until certain minimal structure of the donor substrate. We have demonstrated that the interactions with the nucleotide moiety and the $\beta-\mathrm{PO}_{4}$ are important to stabilize the active compact conformation in which the catalysis occurs. The intrinsic flexibility of PimA might be finely tuned and guided by the donor substrate structural determinants having an impact ultimately in its catalytic activity rate. This study provides an important contribution toward the understanding of such conformational transition, an activation mechanism, in the framework 
of glycosyl transfer reactions mediated by GT-B enzymes. 


\section{ACKNOWLEDGMENTS}

This work was supported by grants from the European Commission, New Medicine for Tuberculosis, contract LSHP-CT-2005-018923 and More Medicines for Tuberculosis, contract HEALTH-F3-2011-260872 (M.E.G.), and MARIE CURIE Reintegration contract 844905 (B.T.); and the MINECO/FEDER EU contracts SAF2010-19096, BIO2013-49022C2-2-R, BFU2016-77427-C2-2-R, Severo Ochoa Excellence Accreditation SEV-2016-0644 (M.E.G.), MINECO/FEDER EU contract PID2019-104090RB-100 (P.M.), and "Juan de la Cierva Postdoctoral program", contract IJCI-2014-19206 (B.T.). We gratefully acknowledge Dr. Ahmed Haouz, Patrick Weber and Prof. Pedro M. Alzari (Institut Pasteur, Paris, France) for help with robotic crystallization and X-ray crystallography data, and Pedro Arrasate for technical assistance (Biofisika Institute, Spain). This study made use of the Diamond Light Source beamline B21 (Oxfordshire, UK) and ALBA synchrotron beamline BL13-XALOC, funded in part by the Horizon 2020 programme of the European Union, iNEXT (H2020 contract 653706). We gratefully acknowledge all members of the Structural Glycobiology Laboratory for valuable scientific discussions.

\section{CONFLICT OF INTEREST STATEMENT}

None declared. 


\section{REFERENCES}

1. Henzler-Wildman K1, Kern D. 2007. Dynamic personalities of proteins. Nature 450:964-972.

2. Frauenfelder, H.; Sligar, S. G.; Wolynes, P. G. The energy landscape and motions of proteins. Science 1991, 254, 1598-1603.

3. Eisenmesser EZ, Millet O, Labeikovsky W, Korzhnev DM, Wolf-Watz M, Bosco DA, Skalicky JJ, Kay LE, Kern D. 2005. Intrinsic dynamics of an enzyme underlies catalysis. Nature 438:117121.

4. Callender R, Dyer RB. The dynamical nature of enzymatic catalysis. Acc Chem Res. 2015;48(2):407-413.

5. Narayanan SP, Nair DG, Schaal D, Barbosa de Aguiar M, Wenzel S, Kremer W, Schwarzinger S, Kalbitzer HR. 2016. Structural transitions in full-length human prion protein detected by xenon as probe and spin labeling of the N-terminal domain. Sci Rep. 6:28419.

6. Torbeev VY, Raghuraman H, Hamelberg D, Tonelli M, Westler WM, Perozo E, Kent SB. 2011. Protein conformational dynamics in the mechanism of HIV-1 protease catalysis. Proc Natl Acad Sci U S A. 108:20982-20987.

7. Korduláková J, Gilleron M, Mikusová K, Puzo G, Brennan PJ, Gicquel B, Jackson M. 2002. Definition of the first mannosylation step in phosphatidylinositol mannoside synthesis. PimA is essential for growth of mycobacteria. J. Biol. Chem. 277:31335-31344.

8. Guerin ME, Korduláková J, Alzari PM, Brennan PJ, Jackson M. 2010. Molecular basis of phosphatidyl-myo-inositol mannoside biosynthesis and regulation in mycobacteria. J. Biol. Chem. 285:33577-33583.

9. Boldrin F, Ventura M, Degiacomi G, Ravishankar S, Sala C, Svetlikova Z, Ambady A, Dhar N, Kordulakova J, Zhang M, Serafini A, Vishwas VG, Kolly GS, Kumar N, Palù G, Guerin ME, Mikusova K, Cole ST, Manganelli R. 2014. The phosphatidyl-myo-inositol mannosyltransferase PimA is essential for Mycobacterium tuberculosis growth in vitro and in vivo. J. Bacteriol. 196:3441-3451.

10. Guerin ME, Kaur D, Somashekar BS, Gibbs S, Gest P, Chatterjee D, Brennan PJ, Jackson M. 2009. New insights into the early steps of phosphatidylinositol mannoside biosynthesis in mycobacteria: PimB' is an essential enzyme of Mycobacterium smegmatis. J. Biol. Chem. 284:25687-25696.

11. Guerin ME, Kordulakova J, Schaeffer F, Svetlikova Z, Buschiazzo A, Giganti D, Gicquel B, Mikusova K, Jackson M, Alzari PM. 2007. Molecular recognition and interfacial catalysis by the essential phosphatidylinositol mannosyltransferase PimA from mycobacteria. J. Biol. Chem. 282:20705-20714.

12. Lairson LL, Henrissat B, Davies GJ, Withers SG. 2008. Glycosyltransferases: structures, functions, and mechanisms. Ann Rev Biochem 77:521-555.

13. Albesa-Jové D, Giganti D, Jackson M, Alzari PM, Guerin ME. (2014) Structure-function relationships of membrane-associated GT-B glycosyltransferases. Glycobiology 24, 108-124.

14. Albesa-Jové D and Guerin ME. 2016. The Conformational Plasticity of Glycosyltransferases. Curr. Opin. Struct. Biol. 40, 23-32.

15. Giganti D, Albesa-Jové D, Urresti S, Rodrigo-Unzueta A, Martínez MA, Comino N, Barilone N, Bellinzoni M, Chenal A, Guerin ME, Alzari PM. 2015. Secondary structure reshuffling modulates glycosyltransferase function at the membrane. Nat. Chem. Biol. 11:16-18.

16. Rodrigo-Unzueta A, Martínez MA, Comino N, Alzari PM, Chenal A, and Guerin ME. 2016. Molecular basis of membrane association by the phosphatidylinositol mannosyltransferase PimA from mycobacteria. J. Biol. Chem. 291:13955-13963.

17. Brodhun F, Tittmann K. 2015. Membrane enzymes: transformers at the interface. Nat. Chem. Biol. 11:102-103.

18. Guerin ME, Schaeffer F, Chaffotte A, Gest P, Giganti D, Korduláková J, van der Woerd M, Jackson M, Alzari PM. 2009. Substrate-induced conformational changes in the essential peripheral membrane-associated mannosyltransferase PimA from mycobacteria: implications for catalysis. J. Biol. Chem. 284:21613-21625.

19. Giganti D, Alegre-Cebollada J, Urresti S, Albesa-Jové D, Rodrigo-Unzueta A, Comino N, 
Kachala M, López-Fernández S, Svergun DI, Fernández JM, Guerin ME. 2013. Conformational plasticity of the essential membrane-associated mannosyltransferase PimA from mycobacteria. $J$. Biol. Chem. 288:29797-29808.

20. Kabsch W. 2010. XDS. Acta Crystallogr. Sect. D: Biol. Crystallogr. 66:125-132.

21. McCoy, A.J., Grosse-Kunstleve, R.W., Adams, P.D., Winn, M.D., Storoni, L.C., Read, R.J. 2007. Phaser crystallographic software. J. Appl. Crystallogr. 40:658-674.

22. Adams, P.D., Afonine, P.V., Bunkóczi, G., Chen, V.B., Davis, I.W., Echols, N., Headd. J.J., Hung, L.W., Kapral, G.J., Grosse-Kunstleve, R.W., McCoy, A.J., Moriarty, N.W., Oeffner, R., Read, R.J., Richardson, D.C., Richardson, J.S., Terwilliger, T.C., Zwart, P.H. 2010. PHENIX: a comprehensive Python-based system for macromolecular structure solution. Acta Crystallogr. Sect. D: Biol. Crystallogr. 66:213-221.

23. Winn, M.D., Ballard, C.C., Cowtan, K.D., Dodson, E.J., Emsley, P., Evans, P.R., Keegan, R.M., Krissinel, E.B., Leslie, A.G., McCoy, A., McNicholas, S.J., Murshudov, G.N., Pannu, N.S., Potterton, E.A., Powell, H.R., Read, R.J., Vagin, A., Wilson, K.S. 2011. Overview of the CCP4 suite and current developments. Acta Crystallogr. Sect. D: Biol. Crystallogr. 67:235-242.

24. Emsley, P., Lohkamp, B., Scott, W.G., Cowtan, K. 2010. Features and development of Coot. Acta Crystallogr. Sect. D: Biol. Crystallogr. 66:486-501.

25. Afonine, P.V., Grosse-Kunstleve, R.W., Echols, N., Headd, J.J., Moriarty, N.W., Mustyakimov, M., Terwilliger, T.C., Urzhumtsev, A., Zwart, P.H., Adams, P.D. 2012. Towards automated crystallographic structure refinement with phenix.refine. Acta Crystallogr. Sect. D: Biol. Crystallogr. 68:352-367.

26. Chen, V.B., Arendall, W.B. 3rd, Headd, J.J., Keedy, D.A., Immormino, R.M., Kapral, G.J., Murray, L.W., Richardson, J.S., Richardson, D.C. 2010. MolProbity: all-atom structure validation for macromolecular crystallography. Acta Crystallogr. Sect. D: Biol. Crystallogr. 66:12-21.

27. Konarev PV, Volkov VV, Sokolova AV, Koch MHJ, Svergun DI. 2003. PRIMUS — a Windows PC-based system for small-angle scat- tering data analysis. J. Appl. Crystallogr. 36:1277-1282.

28. Guinier A. 1939. La diffraction des rayon's x aux tres petits angles: application a l'etude de phenomenes ultramicroscopies. Ann. Phys. 12:161-237.

29. Svergun DI. 1992. Determination of the regularization parameter in indirect-transform methods using perceptual criteria. J. Appl. Crystallogr. 25:495-503.

30. Wrabl JO, Grishin NV. 2001. Homology between O-linked GlcNAc transferases and proteins of the glycogen phosphorylase superfamily. J. Mol. Biol. 314:365-374.

31. Ardèvol A, Rovira C. 2011. The molecular mechanism of enzymatic glycosyl transfer with retention of configuration: evidence for a short-lived oxocarbenium-like species. Angew Chem Int Ed Engl. 50:10897-10901.

32. Ardevol A, Rovira C. Reaction mechanisms in carbohydrate-active enzymes: glycoside hydrolases and glycosyltransferases. 2015. Insights from first principles QM/MM molecular dynamics simulations. J. Am. Chem. Soc. 137:7528-7547.

33. Albesa-Jové D, Cifuente JO, Trastoy B, and Guerin ME. 2019. Quick-soaking of crystals reveals unprecedented insights into the catalytic mechanism of glycosyltransferases. Methods Enzymol. 621:261-279.

34. Albesa-Jové D, Mendoza F, Rodrigo-Unzueta A, Gomollón-Bel F, Cifuente JO, Urresti S, Comino N, Gómez H, Romero-García J, Lluch JM, Sancho-Vaello E, Biarnés X, Planas A, Merino P, Masgrau L and Guerin ME. 2015. A native ternary complex trapped in crystal reveals the catalytic mechanism of a retaining glycosyltransferase. Angew Chem Int Ed Engl. 54:98989902.

35. Albesa-Jové D, Romero-García J, Sancho-Vaello E, Contreras F-X, Rodrigo-Unzueta A, Comino N, Carreras-González A, Arrasate P, Urresti S, Biarnés X, Planas A, and Guerin ME. 2017. Structural Snapshots and Loop Dynamics Along the Catalytic Cycle of Glycosyltransferase GpgS. Structure 25:1034-1044.

36. Albesa-Jové D, Sainz-Polo MA, Marina A, Guerin ME. 2017. Structural Snapshots of the Reaction Center of $\alpha 1,3-$ Galactosyltransferase with Native Substrates Support a Conserved Catalytic Mechanism for Retaining Glycosyltransferases. Angew Chem Int Ed Engl. 56:1485314857.

37. Moremen KW, Haltiwanger RS. 2019. Emerging structural insights into glycosyltransferase- 
mediated synthesis of glycans. Nat Chem Biol. 15(9):853-864.

38. Batt SM, Jabeen T, Mishra AK, Veerapen N, Krumbach K, Eggeling L, Besra GS, Fütterer K. 2010. Acceptor substrate discrimination in phosphatidyl-myo-inositol mannoside synthesis: structural and mutational analysis of mannosyltransferase Corynebacterium glutamicum PimB'. $J$. Biol. Chem. 285:37741-37752.

39. Sancho-Vaello E, Albesa-Jové D, Rodrigo-Unzueta A, and Guerin ME. 2017. Structural basis of phosphatidyl-myo-inositol mannosides biosynthesis in mycobacteria. Biochem. Biophys. Acta. $1862,1355-1367$.

40. Vetting MW, Frantom PA, Blanchard JS. 2008. Structural and enzymatic analysis of MshA from Corynebacterium glutamicum: substrate-assisted catalysis. J. Biol. Chem. 283:15834-15844.

41. Frantom PA, Coward JK, Blanchard JS. 2010. UDP-(5F)-GlcNAc acts as a slow-binding inhibitor of MshA, a retaining glycosyltransferase. J. Am. Chem. Soc. 132:6626-6627

42. Errey JC, Lee SS, Gibson RP, Martinez Fleites C, Barry CS, Jung PM, O'Sullivan AC, Davis BG, Davies GJ. 2010. Mechanistic insight into enzymatic glycosyl transfer with retention of configuration through analysis of glycomimetic inhibitors. Angew Chem Int Ed Engl. 49:12341237.

43. Lee SS, Hong SY, Errey JC, Izumi A, Davies GJ, Davis BG. 2011. Mechanistic evidence for a front-side, SNi-type reaction in a retaining glycosyltransferase. Nat. Chem. Biol. 7:631-638.

44. Blanco Capurro JI, Hopkins CW, Pierdominici Sottile G, González Lebrero MC, Roitberg AE, Marti MA. 2017. Theoretical insights into the reaction and inhibition mechanism of metalindependent retaining glycosyltransferase responsible for mycothiol biosynthesis. J Phys Chem $B$ 121(3):471-478.

45. Hu Y, Walker S. Remarkable structural similarities between diverse glycosyltransferases. Chem. Biol. 9(12):1287-1296.

46. Hu Y, Chen L, Ha S, Gross B, Falcone B, Walker D, Mokhtarzadeh M, Walker S. 2003. Crystal structure of the MurG:UDP-GlcNAc complex reveals common structural principles of a superfamily of glycosyltransferases. Proc. Natl. Acad. Sci. USA. 100(3):845-849.

47. Buschiazzo A, Ugalde JE, Guerin ME, Shepard W, Ugalde RA, Alzari PM. 2004. Crystal structure of glycogen synthase: homologous enzymes catalyze glycogen synthesis and degradation. EMBO J. 23:3196-3205.

48. Lazarus MB, Jiang J, Gloster TM, Zandberg WF, Whitworth GE, Vocadlo DJ, Walker S. 2012. Structural snapshots of the reaction coordinate for O-GlcNAc transferase. Nat. Chem. Biol. 8:966-968.

49. Breton C, Snajdrova L, Jeanneau C, Koca J, Imberty A. 2006. Structures and mechanisms of glycosyltransferases. Glycobiology 16:29R-37R. 


\section{UniProt ID A0QWG6}




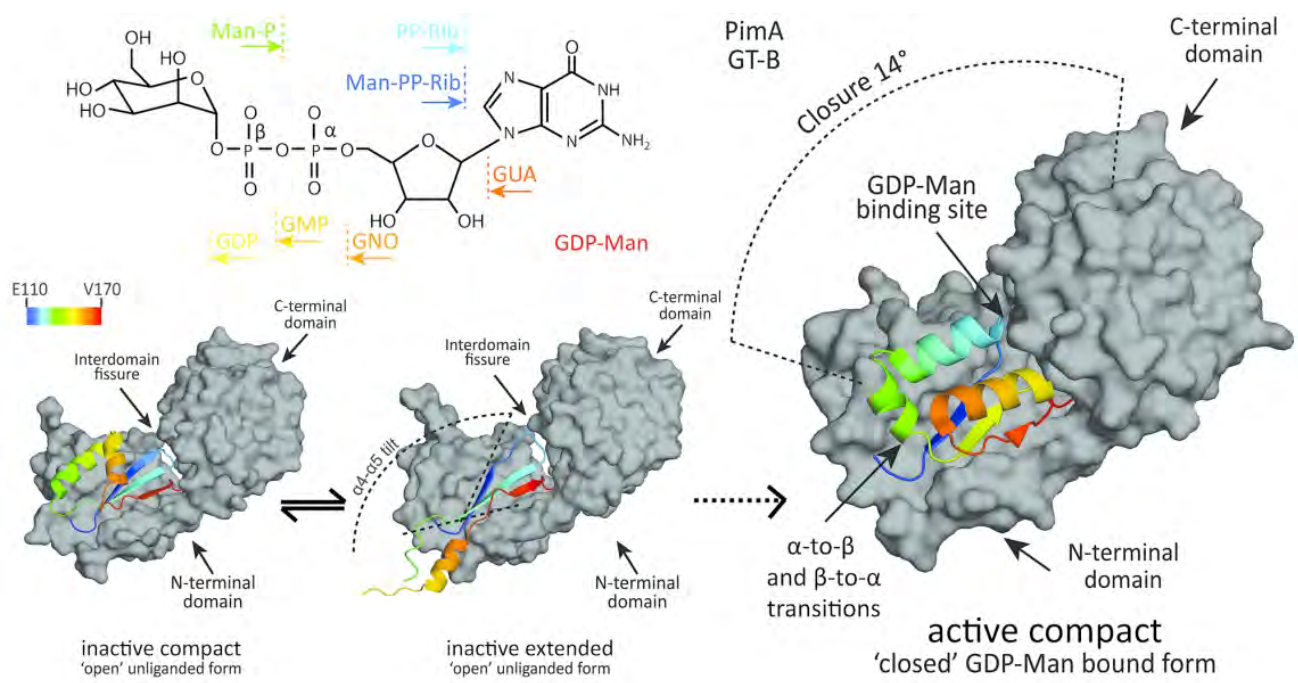

